\title{
Anti-proliferative effect of an analogue of the LL-37 peptide in the colon cancer derived cell line HCT116 $\mathrm{p53}^{+/+}$and $\mathrm{p53}^{-/-}$
}

\author{
KENGO KURODA $^{1}$, TOMOKAZU FUKUDA ${ }^{2}$, HIROSHI YONEYAMA ${ }^{1}$, \\ MASAFUMI KATAYAMA ${ }^{2}$, HIROSHI ISOGAI ${ }^{3}$, KAZUHIKO OKUMURA ${ }^{4}$ and EMIKO ISOGAI ${ }^{1}$ \\ ${ }^{1}$ Laboratory of Animal Microbiology, ${ }^{2}$ Laboratory of Animal Breeding and Genetics, \\ Graduate School of Agricultural Science, Tohoku University, Sendai 981-8555; ${ }^{3}$ Animal Research \\ Center, Sapporo Medical University, Sapporo 060-8556; ${ }^{4}$ Department of Oral and Maxillofacial Surgery, \\ School of Dentistry, Health Sciences University of Hokkaido, Hokkaido 061-0293, Japan
}

Received February 16, 2012; Accepted March 20, 2012

DOI: $10.3892 /$ or.2012.1876

\begin{abstract}
Antimicrobial peptides of the cathelicidin family are found in many mammalian species, and are focused on various effects other than antimicrobial action. In this study, we evaluated the anti-proliferative effect of an analogue peptide, FF/CAP18, derived from an endogenous cathelicidin family member against the colon cancer cell line HCT116. FF/ CAP18 significantly decreased the proliferation of HCT116 cells in a dose-dependent fashion. Furthermore, the treatment of HCT116 with FF/CAP18 caused loss of mitochondrial membrane potential, and resulted in the immunoreactivity to the single-strand DNA antibody, suggesting the early stage of apoptosis. Interestingly, the anti-proliferative effect of FF/CAP18 was constant regardless of the genotype of p53 (wild-type and p53 mutant type HCT116 cells). Therefore, the signaling pathway of p53 is not involved in the growth suppression effect of the cathelicidin analogue peptide. These results indicate that the treatment of certain types of cancer cells with FF/CAP18 may increase the sensitivity of the chemotherapeutic reagents, which might relate to the reduction of the side effects.
\end{abstract}

\section{Introduction}

Antimicrobial peptides of cathelicidin family are found in many mammalian species, such as human being, bovine and swine (1). As the role of endogenous hCAP18/LL-37 (human cationic antimicrobial peptide of $18 \mathrm{kDa}$ ), the innate immunity of this peptide to prevent the infection was previously reported

Correspondence to: Dr Emiko Isogai, Laboratory of Animal Microbiology, Graduate School of Agricultural Science, Tohoku University, 1-1 Amamiya, Tsutsumidori, Aoba-ku, Sendai 981-8555, Japan

E-mail: emiko@bios.tohoku.ac.jp

Key words: LL-37, colon cancer, p53, analogue peptide, antiproliferative effect
(2). The antimicrobial peptide, hCAP18/LL-37 is expressed in various types of organs, such as leucocytes (3), myelocytes (4), testis (5), skin (6), nasal mucosa (7), saliva (8), and the gastrointestinal tract (9). The protein expression and following secretion of the hCAP18/LL-37 result in the involvement of this peptide in the body fluids, such as serum, urine and sweat. The existence of hCAP18/LL-37 in these body fluids is expected to have a biological significance to prevent the bacterial proliferation, and following infection at the local tissues.

In addition to the anti-infectious effect, the anti-proliferative effect of analogue peptide of hCAP18/LL-37 was reported to induce apoptosis against the human squamous cell carcinoma cell line, SAS-H1 (10). Although the antimicrobial peptide is expected to induce the pore formation on the cellular membrane $(11,12)$, the detailed mechanism is poorly understood as to how hCAP18/LL-37 induces the anti-proliferative effect to the malignant cancer cells. In a previous study, the analogue peptide of hCAP18/LL-37, which

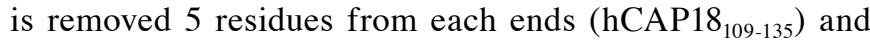
has two amino acid substitutions from the original hCAP18/ LL-37, was reported to have stronger anti-proliferative effect, compared with hCAP18 ${ }_{109-135}(10)$. The anti-proliferative effect of hCAP18 and its analogue peptide against the squamous cell carcinoma cell line (SAS-H1) led us to form the hypothesis that these peptides might be effective in other types of cancer, such as colon cancer.

Colon cancer is classified as one of the major cancers in recent years. The 5-year relative survival rate is around $90 \%$, when detected at early stage of carcinogenesis (stage I). In the late stage, such as metastatic stage, the average of median survival duration is 5-6 months, indicating the high lethality of colon cancer (13). The effectiveness of chemotherapeutic regents is very different among the multiple types of cancers $(14,15)$. This situation guided us to evaluate whether hCAP18/ LL-37 and its analogue peptides are effective in preventing cell proliferation, in the colon cancer derived cell line HCT116. The specific reason for selecting HCT116 as the material is the existence of its genetic mutant of p53 tumor suppressor gene.

The p53 gene product is known to have a central role for apoptosis, cell cycle arrest, DNA repair, tumor suppression 
and sensitivity to chemotherapy. Due to the huge impact of the p53 protein for the protection against carcinogenesis, p53 is called the 'guardian of genome' (16). Bunz et al established the p53 null mutant with the homologous recombination technique from the wild-type HCT116 (17). Although wildtype of HCT116 still keeps the role of the p53, the null mutant lost the function of the p53 (17). Therefore, we can address the association of the p53 gene to the specific phenomenon, when we compared the response of the p53 wild and null

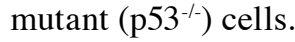

In this study, we exposed the hCAP18/LL-37 and its analogue peptides to HCT116 cells, and evaluated the efficacy to prevent proliferation. Moreover, we compared the effects of peptides among the p53 wild and p53 $3^{--}$HCT 116 cells. Our study would explore the effectiveness of the anti-microbial peptide, hCAP18/LL-37, in colon cancer, and addresses the role of p53 gene on its anti-proliferative effect.

\section{Materials and methods}

Cell culture and reagents. The human colon cancer derived cell line, HCT116, and the isogenic HCT116 $553^{-/}$cells were kindly provided by Dr Bert Vogelstein (The Johns Hopkins University, Baltimore, MD, USA) (17). Cells were cultured in Dulbecco's modified Eagle's medium (DMEM) (Nakarai Tesque, Inc., Kyoto, Japan) with 10\% PBS (Invitrogen, Carlsbad, CA, USA), $1 \%$ antibiotic-antimycotic mixed stock solution (Nakarai Tesque, Inc., Kyoto, Japan) at $37^{\circ} \mathrm{C}$ in $5 \%$ $\mathrm{CO}_{2}$. All cells were kept under the exponential growth condition, and used as the experimental materials.

Peptides. Primary structure of the original hCAP18/LL-37 is represented as LLGDFFRKSKEKIGKEFKRIVQRIKDFLRNLVPRTES (18). The hCAP18 $8_{109-135}$ is the peptide of $27 \mathrm{mer}$, which resulted from the removal of the first and last five amino acids from hCAP18/LL-37. To enhance the anti-microbial activity, FF/CAP18 was designed by replacement of glutamic acid and lysine residue with phenylalanine (FRKSKEKIGKFFKRIVQRIFDFLRNLV) of hCAP18 ${ }_{109-135}$.

Cell growth assay. HCT116 and HCT116 $\mathrm{p} 53^{--}$cells were seeded at $5.0 \times 10^{5}$ cells per 48 -well plate (Falcon, Franklin Lakes, NJ, USA) with complete culture medium, and incubated at $37^{\circ} \mathrm{C}$ in $5 \% \mathrm{CO}_{2}$. Twenty-four hours later, medium was changed with or without FF/CAP18 peptide (total concentration was $10 \mu \mathrm{g} / \mathrm{ml}$ or $40 \mu \mathrm{g} / \mathrm{ml}$ ) in the $250 \mu \mathrm{l}$ complete culture medium. The cells were stained with trypan blue (Invitrogen), and the cell concentration was counted by Countess Automatic Cell Counter (Invitogen) at 24, 48, 72 and $96 \mathrm{~h}$ after the exposure of the peptides.

MitoCapture $^{T M}$ assay. Disruption of mitochondrial transmembrane potential is one of the earliest intracellular events that occur following induction of apoptosis (19). This transmembrane change can be visualized using MitoCapture (Mitochondrial Apoptosis Detection kit, MBL, Nagoya, Japan), under the unfixed cell condition. Mitocapture ${ }^{\mathrm{TM}}$ assay was performed according to manufacturer's instructions. The signal of the MitoCapture was detected by a fluorescence microscope (FSX100, Olympus, Tokyo, Japan).
Immunohistochemistry for single strand (ss) DNA. The genomic DNA causes fragmentation at the time of apoptosis due to the cleavage of the DNase I, which is activated by caspases. To detect the DNA fragmentation and following apoptosis, we conducted immunohistochemical staining of single strand DNA (ss-DNA) for peptide treated cells. The immunohistochemical study was performed on cells prepared by $4 \%$ paraformaldehyde solution in PBS after incubation in a 48-well plate with or without FF/CAP18. The fixed cells were washed with PBS, incubated with $0.5 \%$ Triton X-PBS solution for $30 \mathrm{~min}$ on ice for permeabilization. As the positive control for the DNA fragmentation, the cells were treated with the recombinant DNaseI (Takara Bio Inc., Shiga, Japan) at $37^{\circ} \mathrm{C}$ in $5 \% \mathrm{CO}_{2}$ for $1 \mathrm{~h}$. As the negative control, cells treated with the same volume of PBS was used. After PBS wash, the cells were treated with 2\% normal goat serum in PBS as blocking buffer for $1 \mathrm{~h}$ at room temperature. Anti ss-DNA Rabbit IgG Affinity Purify (Immuno-Biological Laboratories Co., Ltd., Gunma, Japan) was used as first antibody. The samples were exposed to $0.6 \mu \mathrm{g} / \mathrm{ml}$ of antibody at $4^{\circ} \mathrm{C}$ overnight. Cells were washed with PBS for 5 min 3 times, treated with Alexa Fluor 488 (Invitrogen) as second antibody for $2 \mathrm{~h}$ at room temperature under protection from light. The nuclei were visualized by staining with $1 \mu \mathrm{g} / \mathrm{ml}$ of the 4',6-diamidino-2-phenylindole (DAPI) (Dojindo Laboratories, Kumamoto, Japan). The staining feature was detected by a fluorescence microscope system (FSX100, Olympus).

Statistical test. In this study, all of the experiments were carried out with at least triplicated samples. Means and standard errors were calculated. The statistical significance was evaluated with the Student's t-test. A P-value $<0.05$ was considered statistically significant.

\section{Results}

Effect of LL-37 and FF/CAP18 on HCT116 growth. To investigate the effect of cathelicidin family anti-microbial peptides, we exposed LL-37 and the analogue FF/CAP18, to the colon cancer HCT116 cells. The effect to the cell growth was evaluated at $24,48,72$ and $92 \mathrm{~h}$ at $40 \mu \mathrm{g} / \mathrm{ml}$ concentration of the peptide. As shown in Fig. 1A, a relatively slower cell growth (FF/CAP18 treated group) was observed at both 72 and $96 \mathrm{~h}$. However, the effect of the LL-37 was relatively less when it is compared with that of FF/CAP18 (Fig. 1A). We considered that cathelicidin peptides have a potential to suppress the cell growth of the colon cancer cells.

As the next experiments, we detected the detailed antiproliferative effect of FF/CAP18 to the HCT116 cell line, at the multiple dose and time points. We could not see any anti-proliferative effect of FF/CAP18 until $48 \mathrm{~h}$ (Fig. 1B). At $72 \mathrm{~h}$ cell number treated with 10 and $40 \mu \mathrm{g} / \mathrm{ml} \mathrm{FF} / \mathrm{CAP} 18$ was significantly low $(\mathrm{P}<0.05)$, when compared with that of non-treated cells (control). At $96 \mathrm{~h}$, the cell number treated with $40 \mu \mathrm{g} / \mathrm{ml}$ of FF/CAP18 was significantly low $(\mathrm{P}<0.05)$. From these results, we concluded that FF/CAP18 has growth inhibition ability to the HCT116 from $10 \mu \mathrm{g} / \mathrm{ml}$. Furthermore, the effect of the FF/CAP18 to HCT116 was dose-dependent to the peptide concentration. To estimate the cell condition, the cell morphology of the peptide treated cells was compared 
A

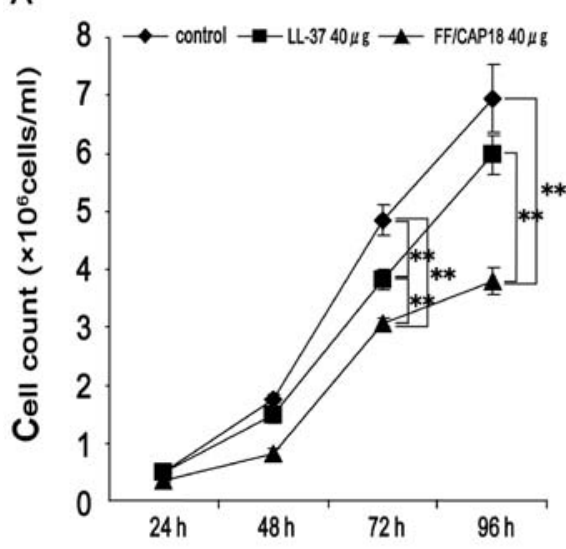

B

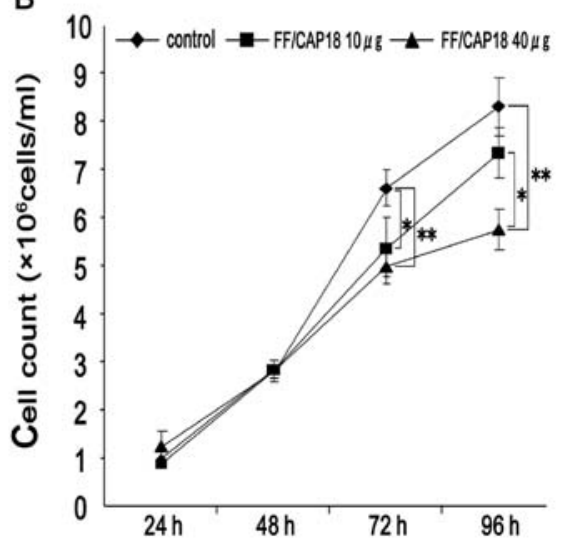

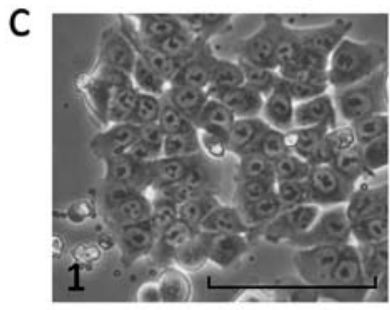

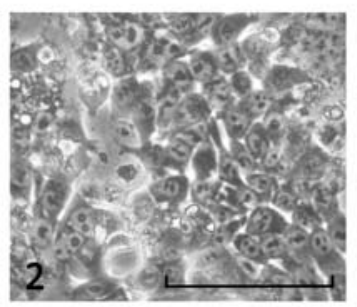

Figure 1. Anti-proliferative effect of cathelicidin family peptide, LL-37 and FF/CAP18 on the colon cancer derived cell line, HCT116. (A) Cells were treated with LL-37 or FF/CAP18 at $40 \mu \mathrm{g} / \mathrm{ml}$ for $96 \mathrm{~h}$ at $37^{\circ} \mathrm{C}$. Each time point contains $4-5$ duplications, and the mean value and SE are shown. The asterisks indicate the statistical significance. ${ }^{* *} \mathrm{P}<0.01$. (B) The dose-dependent anti-proliferative effect of FF/CAP18 on the HCT116. Each time point contains 6 - 8 duplications, and the mean value and SD are shown. The asterisks indicate the statistical significance. ${ }^{* *} \mathrm{P}<0.01,{ }^{*} \mathrm{P}<0.05$. Note that $40 \mu \mathrm{g} / \mathrm{ml}$ of $\mathrm{FF} / \mathrm{CAP} 18$ showed significant impact to the cell growth of HCT116. (C) Morphology of HCT116 cells with (1) or without FF/CAP18 (2). Bar, $100 \mu \mathrm{m}$.
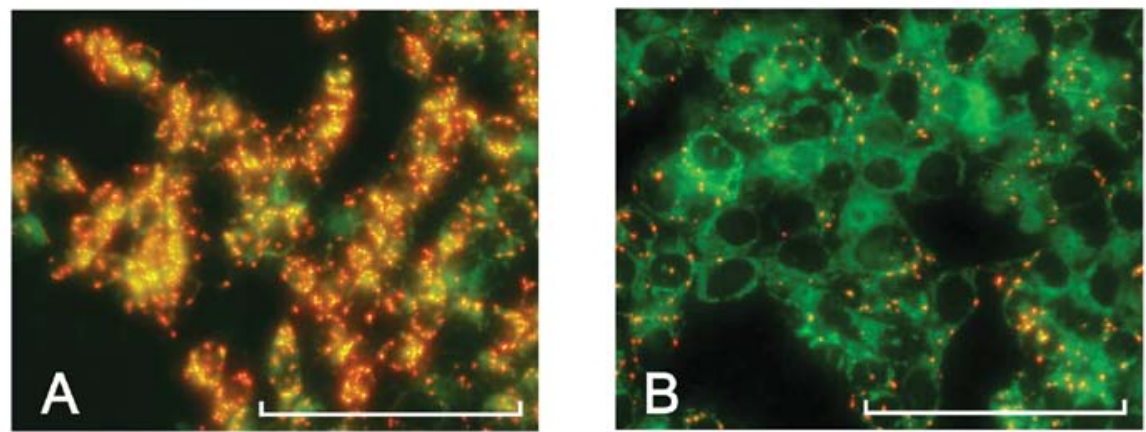

Figure 2. The detection of the disruption of mitochondria membrane with the mitocapture method. (A) The detection of the red fluorescence deposit on the mitochondria in wild-type HCT116 cells. (B) The results of FF/CAP18 treated HCT116. Note that remarkable reduction of the red fluorescence was observed. Bar, $100 \mu \mathrm{m}$.

with that of the control cells (Fig. 1C). Although the growth suppression was obvious in the peptide treated cells, we did not see remarkable change on the morphology of the cells (Fig. 1C).

Loss of mitochondrial membrane potential. Loss of mitochondrial membrane potential is associated with early stage of apoptosis. In present study, MitoCapture ${ }^{\mathrm{TM}}$ is used for detection of this stage. MitoCapture ${ }^{\mathrm{TM}}$ is a cationic dye that enables the accumulation of red fluorescence aggregation in the corresponding area of the mitochondria, due to its transmembrane potential. In contrast, in the early stage of apoptosis, the disruption of the mitochondrial trans-membrane potential is one of the intracellular events, therefore, the aggregation of the red fluorescence does not occurs in the cells, which stay in the early stage of apoptosis. Furthermore, mitochondrial depolarization causes a regression in red fluorescence and an increase in green fluorescence due to the presence of the monomeric form of the dye in the cytoplasm.

To address the cell status of the HCT116 cells treated with the FF/CAP18, the MitoCapture ${ }^{\mathrm{TM}}$ was applied to the analysis. As shown in Fig. 2, the control HCT116 cells showed the intense red signals, which indicate that the mitochondria of control HCT116 cell is intact (Fig. 2A). In contrast, the cells treated with FF/CAP18 showed decreased signal intensity when compared with the control (Fig. 2B). Furthermore, although it signal was faint, certain cell population at high cell density showed green fluorescence, compared with the control 


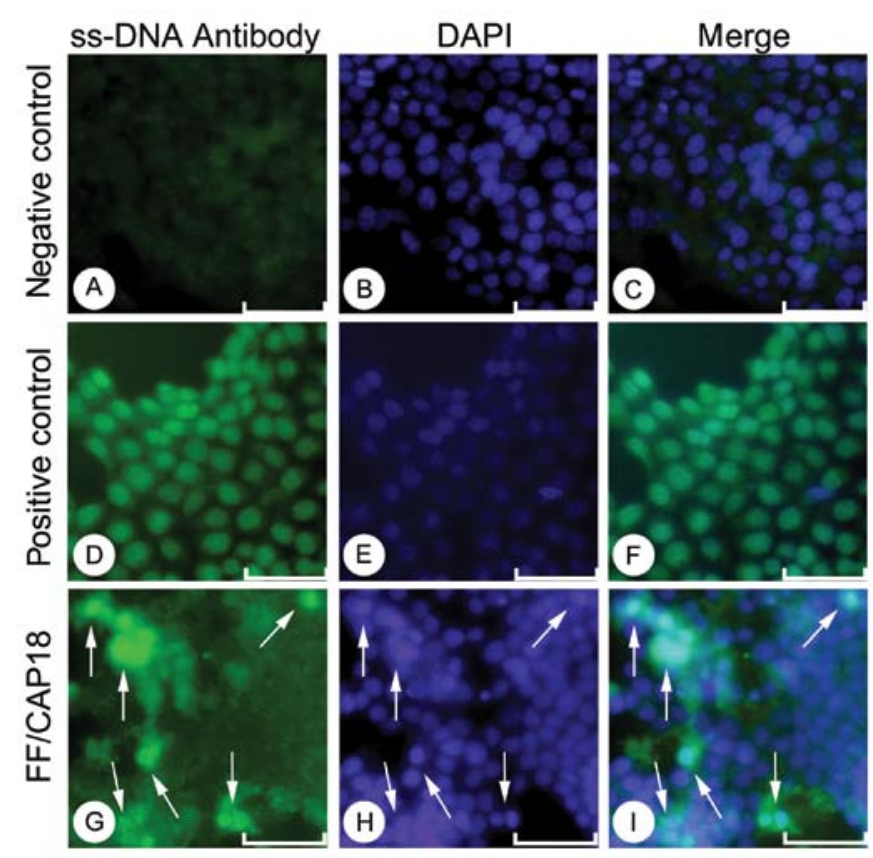

Figure 3. The detection of the genomic DNA fragmentation with single strand DNA antibody. (A-C) No treatment control. Results with anti ss-DNA antibody (A), DAPI staining (B), merge (C) are shown. (D-F) Positive control cells, treated with the DNAse I. (G-I) FF/CAP18 treated cells. Note arrows showing positivity for anti ss-DNA antibody. Bar, $50 \mu \mathrm{m}$.

(data not shown). These results showed that FF/CAP18 causes loss of mitochondrial membrane potential.

$D N A$ fragmentation by $F F / C A P 18$. As the next step from the mitochondrial disruption, the activation of DNase I and fragmentation of the genomic DNA occurs in the process of apoptosis. To detect the DNA fragmentation, we carried out the detection with the anti ss-DNA antibody. In a previous study, the detection of ss-antibody was more sensitive in detecting apoptotic cells, and with low background, and high specificity was indicated, when compared with the TUNEL method (20).

The immunostaining of anti ss-DNA antibody showed the intense positive staining on the nuclei in the positive control cells. For the positive control, we used the HCT116 cells treated with DNase I. The almost no signal in the nuclei in the non-treatment cells (Fig. 3A-C), and the intense staining in the positive control (Fig. 3D-F) facilitate that our detection of the single strand DNA in the nucleus is specific. HCT116 cells, treated with FF/CAP18, showed intense signals in a limited number of cells. The cells showed relatively condensed nuclear, and intense positive reactivity for the anti ss-DNA antibody (Fig. 3G-I, arrows). From these data, although based on a limited number of population, we concluded that FF/CAP18 treatment induces apoptotic cell death in HCT116 cells, which possibly explained the antiproliferative effect.

The anti-proliferative effect of FF/CAP18 in the $p 53^{-/-}$HCT116 cells. The p53 is classified as the genomic guardian due to its nature as the tumor suppressor gene, and loss of function of p53 is commonly observed in around $50 \%$ in various types of

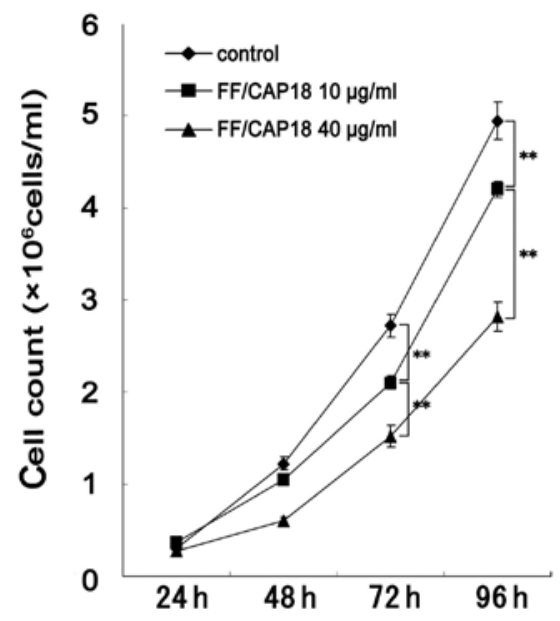

Figure 4. The anti-proliferative effect of FF/CAP18 peptide to $\mathrm{p} 53^{-/-} \mathrm{HCT} 116$ cells. HCT116 p53 $3^{-/-}$cells were treated with or without FF/CAP18 (10 and $40 \mu \mathrm{g} / \mathrm{ml}$ ) in cell culture medium for $96 \mathrm{~h}$ at $37^{\circ} \mathrm{C}$. Each time point contains five duplication, and its mean value and SE were plotted on the graph. The asterisks indicate the statistical significance, ${ }^{* *} \mathrm{P}<0.01,{ }^{*} \mathrm{P}<0.05$. Note that p53 $3^{-/}$HCT116 showed the identical phenotype with the wild-type HCT116.

cancers. The results in the previous section showed that the addition of 10 or $40 \mu \mathrm{g} / \mathrm{ml}$ of FF/CAP18 peptide suppresses the HCT116 cells. We expected that the cellular growth data in p53 mutant of HCT116 would reveal whether p53 tumor suppressor pathways is involved in the anti-proliferative effects of FF/CAP18.

As shown in Fig. 4, the exposure of 10 or $40 \mu \mathrm{g} / \mathrm{ml}$ of FF/ CAP18 peptide significantly suppressed the cell growth of

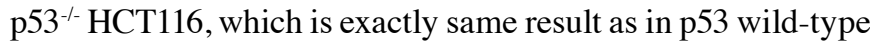
HCT116 cells. The growth of the $\mathrm{p} 53^{-/-}$cells was suppressed at 72 and $96 \mathrm{~h}$ in the 10 or $40 \mu \mathrm{g} / \mathrm{ml}$ treated groups, in a dose-dependent fashion. Therefore, we could not detect any difference in the growth suppression effects of FF/CAP18 peptide among the $\mathrm{p} 53^{-/}$and $\mathrm{p} 53$ wild-type HCT116. We also evaluated the difference among wild-type and p53 mutant of HCT116, in the the MitoCapture assay, and immunohistochemistry of anti ss-DNA antibody, but no differene was detected (data not shown).

\section{Discussion}

In this study, we showed that the hCAP18/LL-37 analogue peptide, FF/CAP18 has anti-proliferative effect on human colon cancer cell HCT116 via inducing depolarization of the partial mitochondrial membrane, which is the earlier stage of apoptosis. The further key finding in this study is that the antiproliferative effect of this peptide is independent from the p53 signaling pathway.

The cationic antimicrobial peptides, such as BMAP-27, BMAP-28, and $\alpha$-difensin, are known to induce apoptosis of tumor cells through mitochondrial damage (21-23). The hCAP18/LL-37 belongs to the cathelicidine anti-microbial peptide family, which includes BMAP-27 and -28 . The cytoplasmic membranes of bacteria contain many anionic phospholipids, causing a negative charge on the cell surface. The cationic antimicrobial peptides, such as hCAP18/LL-37, and BMAP-27 are expected to have a positive charge. The 
positive charge of the peptide and negative charge of the bacterial membrane is one of the possible explanations, how these peptides preferably bind to the bacteria. After the binding of these peptide to the bacteria, the peptides are expected to form the pore on the cell membrane $(11,12)$ or induce the leaking out of the intracellular molecules, resulting in cell death $(12,18)$. In general, there are several reports that the cancer cells have a high contents of anionic phospholipids in their cell membranes, when compared with that of normal cells $(24,25)$. Based on this concept, the cathelicidine anti-microbial peptides might preferably bind to cancer cells, when compared with normal cells.

The genetic diversity of the malignant cancer is one of the important characteristics. Okumura et al reported that hCAP18 ${ }_{109-135}$ (active domain of hCAP18 synthesized as C-terminal 27 amino acids) and its modified peptide, FF/ CAP18 efficiently suppress cell growth of the squamous cell carcinoma SAS-H1 (10). In brief, Okamura et al reported that both hCAP18 ${ }_{109-135}$ and FF/CAP18 are effective in suppressing the growth of the SAS-H1, but its effect was more obvious in FF/CAP18 (10). In this study, we observed the anti-proliferative effect of FF/CAP18, and almost no effect on cell growth in case of LL-37. FF/CAP18 has modified amino acids to enhance the negative charge of the peptide. The difference of the anti-proliferative effect among the LL-37 and FF/CAP18 might be explained by the grade of the negative charge of the peptide. Furthermore, the results of the current study showed that there is diversity among the multiple cancer cell lines for the anti-proliferative effect of FF/CAP18.

FF/CAP18 treated HCT116 cells showed the slower growth, and showed disruption of the mitochondrial membrane, which can be observed at early stage of apoptosis. Until this stage, squamous cell carcinoma cell line, SAS-H1 and colon cancer derived cell line HCT116, showed very similar results. However, in case of SAS-H1, the DNA fragmentation was detected after gel electrophoresis, indicating that SAS-H1 entered into the late stage of apoptosis. In contrast, in this study, we could not detect the massive fragmentation of the genomic DNA, which was detected by electrophoresis of the genomic DNA. These results suggest that the sensitivity to the peptide is different depending on the nature of each cancer cell line.

For the detection of the apoptotic cells in HCT116, we first tried the detection of the apoptosis cells with the standard TUNEL method. However, an obvious high background in FF/CAP18 treated cells were observed (data not shown) and specific detection with the TUNEL method was difficult. Although the detailed situation is unknown, the non-specific binding of the terminal transferase might occur due to the peptide treatment (data not shown). In contrast, fluorescence staining of anti ss-DNA antibody works well, due to the simple immunostaining. The staining of the ss-DNA antibody was previously reported as the effective method to detect apoptotic cells in cancer tissues (20).

The disruption of mitochondrial membrane in the process of apoptosis is largely affected by the function of the p53 gene. The p53 gene is activated after ultra violet and/or radiation exposure $(26,27)$. Although numerous p53 functions have been studied on carcinogenesis, the induction of the cell cycle arrest at G1S and G2M phase of the cell cycle, is when the cell is exposed to damage $(28,29)$. In this study, the wild-type and p53 mutant of HCT116 cells did not show any difference in response to the FF/CAP18 treatment. Our results are in good agreement with the previous published results in SAS-H1. In brief, Okumura et al reported that the cell death induced by the hCAP18 ${ }_{109-135}$ or FF/CAP18 is independent of the activation of the caspase, which is the major downstream molecule of the p53 dependent apoptosis (10). Although the detailed molecular pathway of the cell death induced by the LL-37 or FF/CAP18 is not clear, we revealed that the anti-proliferative effect of LL-37 or FF/CAP18 is independent from p53 tumor suppressor pathway, with the genetic mutant of HCT116 colon cancer cell line.

In recent years, the anticancer effects of several natural compound-derived peptides or toxin have been reported, such as defensin peptide family from a beetle, Allomyrina dichotoma (30) or the toxin like peptide piericin from cabbage butterfly (31). The anticancer effect of these naturally-derived peptides or toxins is not as strong as the chemotherapeutic reagents, however, the anti-proliferative effect might increase the sensitivity of the chemotherapeutic reagents, which might relate to the reduction of the side effects.

\section{Acknowledgements}

This study was supported by the grant from the Japan Science and Technology Agency (AS231Z0188E). We are grateful for the support and encouragement of all members of the Laboratory of Animal Microbiology, Graduate School of Agricultural Science, Tohoku University, all through this study.

\section{References}

1. Zanetti M, Gennaro R, Scocchi M and Skerlavaj B: Structure and biology of cathelicidins. Adv Exp Med Biol 479: 203-218, 2000.

2. Zanetti M: The role of cathelicidins in the innate host defenses of mammals. Curr Issues Mol Biol 7: 179-196, 2005.

3. Cowland JB, Johnsen AH and Borregaard N: hCAP-18, a cathelin/pro-bactenecin-like protein of human neutrophil specific granules. FEBS Lett 368: 173-176, 1995.

4. Sorensen O, Arnljots K, Cowland JB, Bainton DF and Borregaard N: The human antibacterial cathelicidin, hCAP-18, is synthesized in myelocytes and metamyelocytes and localized to specific granules in neutrophils. Blood 90: 2796-2803, 1997.

5. Agerberth B, Gunne H, Odeberg J, Kogner P, Boman HG and Gudmundsson GH: FALL-39, a putative human peptide antibiotic, is cysteine-free and expressed in bone marrow and testis. Proc Natl Acad Sci USA 92: 195-199, 1995.

6. Frohm M, Agerberth B, Ahangari G, et al: The expression of the gene coding for the antibacterial peptide LL-37 is induced in human keratinocytes during inflammatory disorders. J Biol Chem 272: 15258-15263, 1997.

7. Chen PH and Fang SY: The expression of human antimicrobial peptide LL-37 in the human nasal mucosa. Am J Rhinol 18: 381-385, 2004.

8. Murakami M, Ohtake T, Dorschner RA and Gallo RL: Cathelicidin antimicrobial peptides are expressed in salivary glands and saliva. J Dent Res 81: 845-850, 2002.

9. Bals R, Wang X, Zasloff M and Wilson JM: The peptide antibiotic LL-37/hCAP-18 is expressed in epithelia of the human lung where it has broad antimicrobial activity at the airway surface. Proc Natl Acad Sci USA 95: 9541-9546, 1998.

10. Okumura K, Itoh A, Isogai E, et al: C-terminal domain of human CAP18 antimicrobial peptide induces apoptosis in oral squamous cell carcinoma SAS-H1 cells. Cancer Lett 212: 185-194, 2004.

11. Henzler Wildman KA, Lee DK and Ramamoorthy A: Mechanism of lipid bilayer disruption by the human antimicrobial peptide, LL-37. Biochemistry 42: 6545-6558, 2003.

12. Zasloff M: Antimicrobial peptides of multicellular organisms. Nature 415: 389-395, 2002. 
13. Van Cutsem $\mathrm{E}$ and Geboes $\mathrm{K}$ : The multidisciplinary management of gastrointestinal cancer. The integration of cytotoxics and biologicals in the treatment of metastatic colorectal cancer. Best Pract Res Clin Gastroenterol 21: 1089-1108, 2007.

14. Li Z, Song H, He W, Tian Y and Huang T: In vitro chemosensitivity testing of primary and recurrent breast carcinomas and its clinical significance. J Huazhong Univ Sci Technolog Med Sci 28: 683-687, 2008

15. Yanagawa E, Nishiyama M, Saeki T, et al: Chemosensitivity tests in colorectal cancer patients. Jpn J Surg 19: 432-438, 1989.

16. Lane DP and Lain S: Therapeutic exploitation of the $\mathrm{p} 53$ pathway. Trends Mol Med 8: S38-S42, 2002.

17. Bunz F, Dutriaux A, Lengauer C, et al: Requirement for $\mathrm{p} 53$ and p21 to sustain G2 arrest after DNA damage. Science 282: 1497-1501, 1998

18. Johansson J, Gudmundsson GH, Rottenberg ME, Berndt KD and Agerberth B: Conformation-dependent antibacterial activity of the naturally occurring human peptide LL-37. J Biol Chem 273: 3718-3724, 1998

19. Zamzami N, Marchetti P, Castedo M, et al: Sequential reduction of mitochondrial transmembrane potential and generation of reactive oxygen species in early programmed cell death. J Exp Med 182: 367-377, 1995.

20. Watanabe I, Toyoda M, Okuda J, et al: Detection of apoptotic cells in human colorectal cancer by two different in situ methods: antibody against single-stranded DNA and terminal deoxynucleotidyl transferase-mediated dUTP-biotin nick end-labeling (TUNEL) methods. Jpn J Cancer Res 90: 188-193, 1999.

21. Aarbiou J, Ertmann M, van Wetering S, et al: Human neutrophil defensins induce lung epithelial cell proliferation in vitro. J Leukoc Biol 72: 167-174, 2002.
22. Risso A, Braidot E, Sordano MC, et al: BMAP-28, an antibiotic peptide of innate immunity, induces cell death through opening of the mitochondrial permeability transition pore. Mol Cell Biol 22: 1926-1935, 2002.

23. Risso A, Zanetti M and Gennaro R: Cytotoxicity and apoptosis mediated by two peptides of innate immunity. Cell Immunol 189: 107-115, 1998.

24. Utsugi T, Schroit AJ, Connor J, Bucana CD and Fidler IJ: Elevated expression of phosphatidylserine in the outer membrane leaflet of human tumor cells and recognition by activated human blood monocytes. Cancer Res 51: 3062-3066, 1991.

25. Van Blitterswijk WJ, De Veer G, Krol JH and Emmelot P: Comparative lipid analysis of purified plasma membranes and shed extracellular membrane vesicles from normal murine thymocytes and leukemic GRSL cells. Biochim Biophys Acta 688: 495-504, 1982.

26. Morgan SE and Kastan MB: p53 and ATM: cell cycle, cell death, and cancer. Adv Cancer Res 71: 1-25, 1997.

27. Westphal $\mathrm{CH}$ : Cell-cycle signaling: Atm displays its many talents. Curr Biol 7: R789-R792, 1997.

28. Lowe SW and Sherr CJ: Tumor suppression by Ink4a-Arf: progress and puzzles. Curr Opin Genet Dev 13: 77-83, 2003.

29. Vousden KH and Lu X: Live or let die: the cell's response to $\mathrm{p} 53$. Nat Rev Cancer 2: 594-604, 2002.

30. Miyanoshita A, Hara S, Sugiyama M, et al: Isolation and characterization of a new member of the insect defensin family from a beetle, Allomyrina dichotoma. Biochem Biophys Res Commun 220: 526-531, 1996.

31. Nagaoka I, Hirota S, Yomogida S, Ohwada A and Hirata M: Synergistic actions of antibacterial neutrophil defensins and cathelicidins. Inflamm Res 49: 73-79, 2000. 